\title{
Bundesverdienstkreuz am Bande: Antonia Peters und die Deutsche Gesellschaft Zwangserkrankungen
}

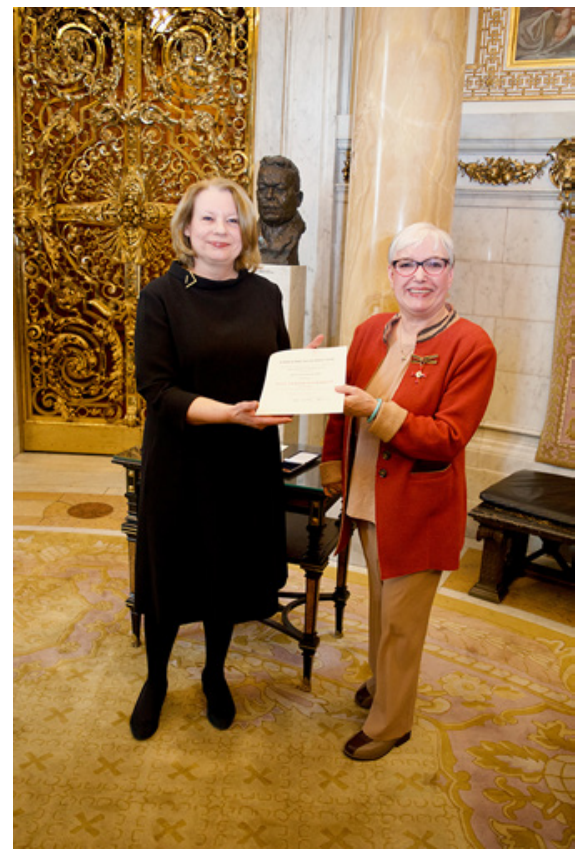

Übergabe des Bundesverdienstkreuzes an Frau Antonia Peters durch Hamburgs Gesundheitssenatorin, Frau Cornelia PrüferStorcks.

Frau Antonia Peters, Vorsitzende der Deutschen Gesellschaft Zwangserkrankungen (DGZ), wurde am 6. März 2020 im Hamburger Rathaus im Rahmen einer Feierstunde das "Bundesverdienstkreuz am Bande" durch die Gesundheitssenatorin, Frau Prüfer-Storcks, überreicht: "Der Bundespräsident verleiht Antonia Peters das Bundesverdienstkreuz am Bande für ihre über 20-jährige
Tätigkeit als Vorstandsmitglied in der 'Deutschen Gesellschaft Zwangserkrankungen e.V.' sowie für ihren langjährigen Einsatz im Verein 'Irre menschlich Hamburg e.V.' Die gelernte Erzieherin trägt maßgeblich zur Prävention und Aufklärung von Zwangserkrankungen bei insbesondere im Bereich der Trichotillomanie" (Pressemitteilung der Hamburger Behörde f. Gesundheit und Verbraucherschutz, 2. März 2020).

Alle über die Jahrzehnte in der DGZ aktiven Betroffenen, Angehörigen, Therapeuten und Forscher sind durch die Auszeichnung für ihre Vorstandsvorsitzende mit geehrt.

Warum ist diese Ehrung auch für die Verhaltenstherapie in Deutschland ein besonderes Ereignis? Für alle, die Antonia Peters noch nicht kennen, im Folgenden dazu einige Informationen.

\section{Antonia Peters und die DGZ - Oder: "Von der Betroffenen zur Macherin"}

1995 gründeten ein Journalist (dpa), ein Professor für Persönlichkeitspsychologie, ein Designer, eine psychologische Verhaltenstherapeutin und ein Professor für Psychiatrie/Verhaltenstherapie, zusammen mit zwei Vertretern einer Pharmafirma, die DGZ und deren Zeitschrift "Z-aktuell". Die berufliche Heterogenität dieses Gremiums rührte aus der Erkenntnis der Beteiligten her, dass Zwangsverhaltensweisen nicht nur unter den psychischen Störungen, sondern auch in der Psyche der Normalbevölkerung wie in gesellschaftlichen Abläufen eine hohe Bedeutung haben. Ziel war von vornherein, die 
DGZ schrittweise in ein Gremium von Betroffenen/Angehörigen und Therapeuten/Forschern umzuwandeln. Das ist, trotz einiger Anlaufschwierigkeiten, auch innerhalb weniger Jahre gelungen (Überblick über die ersten 20 Jahre dieser Entwicklung im entsprechenden Sonderheft von "Z-aktuell", 3/2015).

1998 begann die beeindruckende Entwicklung von Antonia Peters in der DGZ mit ihrem Eintritt als eine von Trichotillomanie (TTM) Betroffene. Bereits 1999 wurde sie als erste Betroffene in den erweiterten Vorstand gewählt, 2001 wurde sie stellvertretende Vorsitzende und seit 2004 ist sie erste Vorsitzende. Diese Entwicklung im DGZ-Vorstand beruhte zum einen sicherlich auf ihren guten Managementfähigkeiten sowohl bezüglich auftretender Konflikte im Leitungsgremium wie auch bezüglich der Organisation der Arbeitsabläufe und insbesondere auch der, zusammen mit dem Gesamtvorstand, über viele Jahre sehr erfolgreichen Öffentlichkeitsarbeit. Besonderes Anliegen war ihr dabei immer, die Kenntnis über ihre eigene Störung, die TTM, mit zu fördern.

Dies geschah auf den von ihr mit organisierten, ersten (1997) und zweiten (2003) deutschen Tagungen zur TTM an der Verhaltenstherapie (VT)-Ambulanz UKE in Hamburg (1997), mit eigenen TTM-Veranstaltungen auf Jahrestagungen der DGZ seit 1999, beim zweiten Internationalen Zwangskongress in Hamburg (1999) und auf Jahrestagungen der DGPPN (seit 2015; seither DGZ auch Mitglied im Trialogischen Forum der DGPPN). Seit 2001 hat sie für mehrere öffentliche und private Fernseh- und Radiosender, Zeitungen wie "Zeit" und "Süddeutsche Zeitung" und Zeitschriften bis hin zu "Bravo" Beiträge geliefert. Zusammen mit Wolf Hartmann, dem Geschäftsführer der DGZ, führte sie bis 2002 bundesweit insgesamt 15 Informationsveranstaltungen zur TTM für Betroffene durch. Daraus entstanden Selbsthilfegruppen in Hamburg (1997), 8 weiteren deutschen Großstädten und in Zürich. Die Hamburger Gruppe entwickelte einen kreativen Info Flyer für Friseure, die ja am "Ort des Geschehens" wirken. 2008 gab sie einen Ratgeber für Betroffene heraus (Neuauflage 2020). Am jährlichen "Tag der offenen Tür" der Klinik für Psychiatrie und Psychotherapie des UKE nimmt sie regelmäßig mit Informationsveranstaltungen zu Zwangsstörungen teil, und über den dort angesiedelten Verein "Irre menschlich" führt sie 2- bis 3-mal jährlich an Schulen Aufklärungsveranstaltungen zu Zwangsstörungen durch. Aufgrund der DGZ-Aktivitäten werden inzwischen auch an fast einem halben Dutzend psychosomatischen und einer Universitätsklinik Spezialangebote für Patienten mit Zwangsstörungen, incl. TTM, vorgehalten. Die DGZ hat ferner eine Liste mit 70 niedergelassenen Verhaltenstherapeuten, die spezifisch auch TTM-Betroffene behandeln. Bei Facebook gibt es 2 TTM- und 2 Zwangs-Gruppen, die Antonia Peters - neben dem Zwangsforum der DGZ - betreut.
Zusätzlich bietet sie seit 20 Jahren regelmäßig Telefonberatung für TTM-Betroffene und deren Angehörige an.

Bundesweit hat sie mit der DGZ Selbsthilfegruppen für Zwangskranke und für Angehörige mitgegründet oder unterstützt und sich diesbezüglich auch besonders vor Ort in Hamburg engagiert. Über ihre Homepage bietet die DGZ seit 25 Jahren Betroffenen und Angehörigen sehr viele hilfreiche Informationen zum Krankheitsbild, zu Tagungen und zu den Selbsthilfegruppen an.

Die besondere Bedeutung einer kontinuierlichen Forschung für die Therapie der Zwangserkrankungen haben die DGZ und ihre Vorsitzende immer durch ihren wissenschaftlichen Beirat unterstrichen, der seit nunmehr 7 Jahren kontinuierlich durch Prof. Ulrich Voderholzer, Schön-Klinik Roseneck und LMU München, geleitet wird.

Last but not least sei Antonia Peters' herausragende Betreuung der seit Gründung der DGZ erscheinenden Zeitschrift "Z-aktuell", die für Betroffene wie Therapeuten gleichermaßen informativ ist, erwähnt.

\section{Antonia Peters - als Betroffene und als Person}

Die TTM trat erstmals 1970, im Alter von 11 Jahren, während einer Kur auf. Das führte 1976 zum Tragen eines Kopftuches, welches 1980 durch eine Perücke ersetzt wurde, mit dem Entschluss 4 Jahre später, die Problemzone nicht mehr zu verstecken. Noch ein Jahr später suchte sie dann erstmals professionelle Hilfe. Da TTM in jenen Jahren in der Öffentlichkeit und unter Therapeuten kaum als Krankheit bekannt war, begann jetzt eine Odyssee durch das Versorgungssystem, in der Frau Peters ihre beeindruckende Willensstärke, Entschlossenheit und Handlungsbereitschaft zeigte und weiterentwickelte:

1985 auf Veranlassung eines Neurologen eine erste ambulante VT, die ihr Selbstvertrauen deutlich stärkte, auf das Symptomverhalten aber nicht einging und dieses nicht beeinflußte. Deshalb auf Veranlassung des Hausarztes 1988 eine stationäre Psychotherapie mit deutlicher Symptomverschlechterung. Danach Hilfesuche außerhalb des psychotherapeutischen Versorgungssystems, die $\mathrm{zu}$ neuen, wichtigen Kontakten $\mathrm{zu}$ anderen Betroffenen führte. Aber erst 1997 kam es über eine erste Studie zur TTM (VT vs. selektive Serotonin-Wiederaufnahmehemmer, SSRI) der VT-Ambulanz des UKE in Hamburg zu einem weiteren Behandlungsversuch. Frau Peters entschied sich für den SSRI - und wurde schon nach kurzer Zeit symptomfrei. Das nutzte sie umgehend zur Intensivierung ihrer Bemühungen um Selbsthilfegruppen für TTM, den Eintritt in die DGZ (1998) und, als erste Betroffene, in deren erweiterten Vorstand (1999). Im gleichen Jahr sorgte sie dafür, dass TTM ein Schwerpunktthema auf der 3. Jahrestagung der DGZ wurde. Es über- 
rascht nicht, dass sie Ende des gleichen Jahres, angesichts all der erfolgreichen Aktivitäten, voller Selbstvertrauen ohne Medikament (und dessen Nebenwirkungen) auskommen wollte. So schnell wie die TTM unter dem SSRI zurückgegangen war, so schnell kam sie jetzt wieder. Nach tiefer, aber kurzer Enttäuschung beschloss Frau Peters, in der VT-Ambulanz nun das VT-Programm mitzumachen (2000). Nach 1,5 Jahren und 50 Einzelsitzungen mit intensiver sowohl Symptom- wie "Ursachen"-Therapie hatte sie sich wieder symptomfrei "gearbeitet" und praktizierte überzeugt und hoch motiviert das Erlernte über anderthalb Jahre in intensiver Selbsthilfe weiter!

Frau Peters hatte in für sie sehr überzeugender Weise ausprobiert, welchen relativen Stellenwert die Medikation, die konsequenten Symptomübungen und die Bewältigung von Risikofaktoren und "Ursachen" bei der Bewältigung ihres Zwangsverhaltens hatte. Sie ist diesbezüglich seitdem überzeugte und überzeugende VT-Ratgeberin für andere Zwangskranke.
Wir können daraus folgern:

VT wirkt nachhaltig - wenn sie als "Hilfe zur Selbsthilfe" aktiv angenommen und auf Augenhöhe abgeschlossen wird - und wenn ihre Inhalte dann anhaltend gelebt werden.

Iver Hand, Hamburg

Ehrenvorsitzender der DGZ

\section{Anhang: Zugang zu den Internetangeboten}

https://www.zwaenge.de/

https://www.facebook.com/Deutsche-Gesellschaft-Zwangserkrankungen-eV-1581683845237905/

https://www.facebook.com/Trichotillomanie-Infostelle-176682676023307/

https://www.facebook.com/groups/Trichotillomanie.Gruppe/ https://www.facebook.com/groups/237556656320649/ https://www.facebook.com/groups/186523751436371/ 